\title{
Nucleophilic Substitution by Grignard Reagents on Sulfur Mustards
}

Journal of Organic Chemistry, 2004

Supporting Information

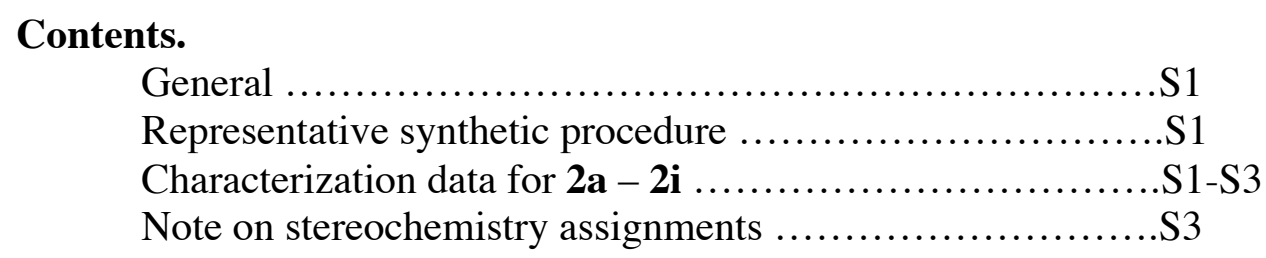

General. Grignard reagents leading to compounds 2a-i where purchased from Aldrich in 0.5-3M solutions in $\mathrm{THF}$ or $\mathrm{Et}_{2} \mathrm{O}$ and were used as received. The remaining organomagnesium reagents were prepared in THF according to literature procedures. Ether and THF were dried by passage through activated alumina or were purchased from Acros. NMR spectra were acquired on a $400 \mathrm{MHz}$ instrument unless otherwise noted.

Representative procedure. 2,6-Diphenyl-9-thiabicyclo[3.3.1]nonane (2a). A two-necked roundbottomed flask equipped with a reflux condenser was flame-dried and charged with $\mathrm{PhMgBr}$ ( $3 \mathrm{M}$ solution in $\mathrm{Et}_{2} \mathrm{O}, 1.775 \mathrm{~mL}, 3.55 \mathrm{mmol}, 2.5$ equiv) and dry toluene ( $8 \mathrm{~mL}$ ) under a stream of $\mathrm{N}_{2}$. 2,6-Dichloro-9-thiabicyclo[3.3.1]nonane was added (300 $\left.\mathrm{mg}, 1.42 \mathrm{mmol}\right)$ and the reaction mixture was heated to a bath temperature of $70{ }^{\circ} \mathrm{C}$. Upon disappearance of starting material (30 minutes; TLC on silica gel, 10:1 hexanes:EtOAc), the reaction was quenched with saturated aqueous ammonium chloride, $\mathrm{Et}_{2} \mathrm{O}$ was added, the phases were separated and the aqueous phase was extracted once more with $\mathrm{Et}_{2} \mathrm{O}$. The combined organic phases were washed with saturated $\mathrm{NaHCO}_{3}$ and brine, and then dried over $\mathrm{MgSO}_{4}$. Filtration and removal of the solvent afforded $408 \mathrm{mg}$ of $\mathbf{2 a}$ as an off-white solid (1.39 mmol, $98 \%$ yield); $\mathrm{mp} 101-103{ }^{\circ} \mathrm{C}$. ${ }^{1} \mathrm{H}$ NMR $\left(400 \mathrm{MHz}, \mathrm{CDCl}_{3}\right): \delta 1.93(\mathrm{~m}, 2 \mathrm{H}), 2.17(\mathrm{~m}, 4 \mathrm{H}), 2.69$ (ddd, 2H, J=27.1, 13.2, $\left.5.9 \mathrm{~Hz}\right), 2.89$ (dd, $2 \mathrm{H}, \mathrm{J}=3.7 \mathrm{~Hz}$ ), $3.67(\mathrm{ddd}, 2 \mathrm{H}, \mathrm{J}=13.2,4.7 \mathrm{~Hz}), 7.27(\mathrm{~m} .2 \mathrm{H}), 7.36(\mathrm{~m}, 8 \mathrm{H}) ;{ }^{13} \mathrm{C}$ NMR $(100$ $\left.\mathrm{MHz}, \mathrm{CDCl}_{3}\right): \quad \delta 26.6,28.0,38.2,48.6,126.5,127.6,128.5,144.8 . \mathrm{HRMS}_{\left(\mathrm{MALDI}^{+} \text {) calcd for }\right.}$ $\mathrm{C}_{20} \mathrm{H}_{23} \mathrm{~S}\left[(\mathrm{M}+\mathrm{H})^{+}\right]: 295.1520$, found 295.1511 .

2,6-di-o-tolyl-9-thiabicyclo[3.3.1]nonane (2b). o-TolylMgBr was used as a $2 \mathrm{M}$ solution in $\mathrm{Et}_{2} \mathrm{O}$. 98\% yield; mp 166-168 ${ }^{\circ} \mathrm{C}$. ${ }^{1} \mathrm{H}$ NMR $\left(400 \mathrm{MHz}, \mathrm{CDCl}_{3}\right): \delta 1.84(\mathrm{~m}, 2 \mathrm{H}), 2.21(\mathrm{~m}, 4 \mathrm{H})$, $2.76(\mathrm{~s}, 6 \mathrm{H}$ ), 2.77 (dd, 2H, J=3.3 Hz), 2.85 (ddd, 2H, J=27.2, 13.6, $6.3 \mathrm{~Hz}$ ), 3.92 (ddd, $2 \mathrm{H}, \mathrm{J}=$ 
13.2, $5.1 \mathrm{~Hz}), 7.20(\mathrm{~m}, 6 \mathrm{H}), 7.41(\mathrm{~d}, 2 \mathrm{H}, \mathrm{J}=7.3 \mathrm{~Hz}) ;{ }^{13} \mathrm{C} \mathrm{NMR}\left(100 \mathrm{MHz}, \mathrm{CDCl}_{3}\right): \delta$ 19.7, 26.7, 28.4, 35.9, 44.4, 125.9, 126.1, 126.3, 130.9, 136.3, 142.7. HRMS (MALDI ${ }^{+}$) calcd for $\mathrm{C}_{22} \mathrm{H}_{27} \mathrm{~S}$ $\left[(\mathrm{M}+\mathrm{H})^{+}\right]: 323.1833$, found 323.1833 .

2,6-di-2-methyl-1-naphthyl-9-thiabicyclo[3.3.1]nonane (2c). The Grignard reagent was prepared according to standard literature procedures with activation of the magnesium by addition of catalytic iodine. $62 \%$ yield; m.p. $178-180{ }^{\circ} \mathrm{C} .{ }^{1} \mathrm{H} \mathrm{NMR}\left(400 \mathrm{MHz}, \mathrm{CDCl}_{3}\right): \delta 1.80$ (d, $6 \mathrm{H}, \mathrm{J}=2.3 \mathrm{~Hz}), 2.09(\mathrm{~m}, 6 \mathrm{H}), 2.48(\mathrm{~m}, 2 \mathrm{H}), 2.72(\mathrm{~m}, 2 \mathrm{H}), 3.13(\mathrm{~m}, 2 \mathrm{H})$. Elemental analysis calcd. for $\mathrm{C}_{16} \mathrm{H}_{26} \mathrm{~S}$ : C, 76.73; $\mathrm{H}, 9.97$. Found: C, 76.66; $\mathrm{H}, 9.56$.

2,6-divinyl-9-thiabicyclo[3.3.1]nonane (2d). VinylMgBr was used as a $1 \mathrm{M}$ solution in THF; most of the THF solvent was removed from the reaction mixture prior to the addition of 1. 69\% yield; clear oil. ${ }^{1} \mathrm{H}$ NMR (400 MHz, $\left.\mathrm{CDCl}_{3}\right): \delta 1.76(\mathrm{~m}, 2 \mathrm{H}), 1.87(\mathrm{~m}, 2 \mathrm{H}), 2.09(\mathrm{~m}, 2 \mathrm{H})$, $2.24(\mathrm{bd}, 2 \mathrm{H}, \mathrm{J}=3.2 \mathrm{~Hz}), 2.70(\mathrm{dd}, 2 \mathrm{H}, \mathrm{J}=6 \mathrm{~Hz}), 2.91(\mathrm{~m}, 2 \mathrm{H}), 4.98(\mathrm{dt}, \mathrm{J}=10.6,1.8 \mathrm{~Hz}, 2 \mathrm{H})$, $5.00(\mathrm{dt}, \mathrm{J}=17.3,1.8 \mathrm{~Hz}, 2 \mathrm{H}), 5.77$ (ddd, $\mathrm{J}=17.3,10.6,7.0 \mathrm{~Hz}, 2 \mathrm{H}) ;{ }^{13} \mathrm{C} \mathrm{NMR}(100 \mathrm{MHz}$, $\left.\mathrm{CDCl}_{3}\right): \delta 27.0,27.4,36.5,45.4,113.9,142.2$. HRMS $\left(\mathrm{MALDI}^{+}\right)$calcd for $\mathrm{C}_{12} \mathrm{H}_{19} \mathrm{~S}\left[(\mathrm{M}+\mathrm{H})^{+}\right]$: 195.1202, found 195.1196 .

2,6-allyl-9-thiabicyclo[3.3.1]nonane (2e). AllylMgBr was used as a $1 \mathrm{M}$ solution in $\mathrm{Et}_{2} \mathrm{O}$. 90\% yield; clear oil. ${ }^{1} \mathrm{H}$ NMR $\left(400 \mathrm{MHz} \mathrm{CDCl}_{3}\right): \delta 1.59(\mathrm{~m}, 2 \mathrm{H}), 1.75(\mathrm{~m}, 2 \mathrm{H}), 2.03(\mathrm{~m}, 4 \mathrm{H})$, $2.15(\mathrm{~m}, 2 \mathrm{H}), 2.26(\mathrm{~m}, 2 \mathrm{H}), 2.65(\mathrm{dd}, 2 \mathrm{H}, \mathrm{J}=3.52 \mathrm{~Hz}), 5.00(\mathrm{~m}, 4 \mathrm{H}), 5.76(\mathrm{~m}, 2 \mathrm{H}) ;{ }^{13} \mathrm{C}$ NMR $\left(100 \mathrm{MHz}, \mathrm{CDCl}_{3}\right): \delta 27.7,29.1,35.6,40.7,41.5,115.9,136.6 . \quad \mathrm{HRMS}\left(\mathrm{MALDI}^{+}\right)$calcd for $\mathrm{C}_{14} \mathrm{H}_{23} \mathrm{~S}\left[(\mathrm{M}+\mathrm{H})^{+}\right]: 223.1520$, found 223.1509.

2,6-dipropynyl-9-thiabicyclo[3.3.1]nonane (2f). PropynylMgBr was used as a $0.5 \mathrm{M}$ solution in THF; most of the THF solvent was removed from the reaction mixture prior to the addition of $1.97 \%$ yield; m.p. $115-117{ }^{\circ} \mathrm{C}$. ${ }^{1} \mathrm{H}$ NMR $\left(400 \mathrm{MHz}, \mathrm{CDCl}_{3}\right): \mathrm{d} 1.80(\mathrm{~d}, 6 \mathrm{H}, \mathrm{J}=2.3$ $\mathrm{Hz}), 2.09(\mathrm{~m}, 6 \mathrm{H}), 2.48(\mathrm{~m}, 2 \mathrm{H}), 2.72(\mathrm{~m}, 2 \mathrm{H}), 3.13(\mathrm{~m}, 2 \mathrm{H}) ;{ }^{13} \mathrm{C} \mathrm{NMR}\left(100 \mathrm{MHz}, \mathrm{CDCl}_{3}\right): \delta 3.4$, 27.5, 28.7, 34.5, 35.3, 76.9, 81.9. HRMS (MALDI ${ }^{+}$) calcd for $\mathrm{C}_{14} \mathrm{H}_{19} \mathrm{~S}\left[(\mathrm{M}+\mathrm{H})^{+}\right]: 219.1202$, found 219.1205 .

2,6-diethynyl-9-thiabicyclo[3.3.1]nonane (2g). EthynylMgBr was used as a $0.5 \mathrm{M}$ solution in THF; most of the THF solvent was removed from the reaction mixture prior to the addition of 61. $30 \%$ yield; m.p. $66-67^{\circ} \mathrm{C}$. ${ }^{1} \mathrm{H}$ NMR $\left(400 \mathrm{MHz}, \mathrm{CDCl}_{3}\right): \delta 2.06(\mathrm{~m}, 2 \mathrm{H}), 2.17(\mathrm{~d}, 2 \mathrm{H}, \mathrm{J}=2.6$ $\mathrm{Hz}), 2.51(\mathrm{~m}, 2 \mathrm{H}), 2.81(\mathrm{~m}, 2 \mathrm{H}), 3.21(\mathrm{~m}, 2 \mathrm{H}) ;{ }^{13} \mathrm{C}$ NMR $\left(100 \mathrm{MHz}, \mathrm{CDCl}_{3}\right): \delta 27.3,28.3,34.1$, 34.9, 69.9, 84.6. HRMS (MALDI ${ }^{+}$) calcd for $\mathrm{C}_{12} \mathrm{H}_{5} \mathrm{~S}\left[(\mathrm{M}+\mathrm{H})^{+}\right]$: 191.0889, found 191.0888 . 
2,6-dipentyl-9-thiabicyclo[3.3.1]nonane (2h). $n$-PentylMgBr was used as a $2 \mathrm{M}$ solution in $\mathrm{Et}_{2} \mathrm{O} .99 \%$ yield; clear oil. ${ }^{1} \mathrm{H} \mathrm{NMR}\left(400 \mathrm{MHz}, \mathrm{CDCl}_{3}\right): \delta 0.88(\mathrm{t}, 6 \mathrm{H}, \mathrm{J}=6.9 \mathrm{~Hz}), 1.20(\mathrm{~m}$, 16H), 1.55 (ddd, $2 \mathrm{H}, \mathrm{J}=27.0,12.9,6.2 \mathrm{~Hz}$ ), $1.73\left(\mathrm{~m}, 2 \mathrm{H}\right.$ ), 2.12 (m, 6H), 2.64 (dd, $2 \mathrm{H} \mathrm{J}=3.5$ ); ${ }^{13} \mathrm{C}$ NMR $\left(100 \mathrm{MHz}, \mathrm{CDCl}_{3}\right): \delta 14.0,22.6,26.4,27.9,29.4,31.9,36.1,36.2,41.4 . \mathrm{HRMS}\left(\mathrm{MALDI}^{+}\right.$) calcd for $\mathrm{C}_{18} \mathrm{H}_{35} \mathrm{~S}\left[(\mathrm{M}+\mathrm{H})^{+}\right]$: 283.2459 , found 283.2458 .

2,6-dimethyl-9-thiabicyclo[3.3.1]nonane (2i). MethylMgBr was used as a $3 \mathrm{M}$ solution in $\mathrm{Et}_{2} \mathrm{O} .82 \%$ yield; clear oil. ${ }^{1} \mathrm{H} \mathrm{NMR}\left(400 \mathrm{MHz}, \mathrm{CDCl}_{3}\right): \delta 0.97(\mathrm{~d}, 6 \mathrm{H}, \mathrm{J}=6.74 \mathrm{~Hz}), 1.60(\mathrm{~m}, 4 \mathrm{H})$, $2.07(\mathrm{~m}, 2 \mathrm{H}), 2.22(\mathrm{~m}, 2 \mathrm{H}), 2.33(\mathrm{~m}, 2 \mathrm{H}), 2.52(\mathrm{~m}, 2 \mathrm{H}) ;{ }^{13} \mathrm{C} \mathrm{NMR}\left(100 \mathrm{MHz}, \mathrm{CDCl}_{3}\right): \delta 21.9$, 27.6, 30.8, 36.7, 37.6. HRMS (MALDI ${ }^{+}$) calcd for $\mathrm{C}_{10} \mathrm{H}_{19} \mathrm{~S}\left[(\mathrm{M}+\mathrm{H})^{+}\right]$: 171.1202, found 171.1202.

Note on stereochemistry assignments. Both the IR bands reported in Table 1 and the NMR spectra of these products are useful for confirming relative configuration. The coupling pattern for $\mathrm{H}^{2}$ is characteristic, and the appearance of this resonance changes dramatically upon substitution with inversion instead of retention. Examples of the latter have been obtained for the oxygen analogues of the type pictured here.

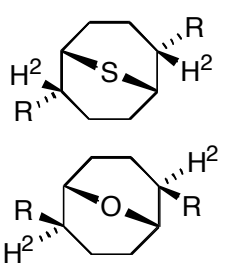

X-ray Crystallography. Details are given in the two .cif files available at http://pubs.acs.org, or from the authors (mgfinn@scripps.edu). 